\title{
Quantitative Cross-Polarization Optical Coherence Tomography Detection of Infiltrative Tumor Margin in a Rat Glioma Model: a Pilot Study
}

DOI: 10.17691/stm2018.10.1.01

Received October 8, 2017

E.B. Kiseleva, PhD, Researcher, Laboratory for Studies of Optical Structure of Biotissues,

Institute of Biomedical Technologies';

K.S. Yashin, MD, Neurosurgeon, Institute of Traumatology and Orthopedics ${ }^{1}$; Junior Researcher, Laboratory of Super-Resolution Microscopy and Gene Technologies, Institute of Biomedical Technologies';

A.A. Moiseev, PhD, Senior Researcher, Laboratory of High-Sensitivity Optical Measurements;

L.B. Snopova, DSc, Head of the Morphology Department, Central Scientific Research Laboratory';

G.V. Gelikonov, PhD, Head of the Laboratory of High-Sensitivity Optical Measurements²;

I.A. Medyanik, MD, DSc, Senior Researcher, Microneurosurgery Group, Institute of Traumatology and Orthopedics ${ }^{1}$;

L.Ya. Kravets, MD, DSc, Professor, Chief Researcher, Microneurosurgery Group, Institute of Traumatology and Orthopedics';

N.N. Karyakin, MD, DSc, Rector';

I.A. Vitkin, PhD, Professor, Departments of Medical Biophysics ${ }^{3}$ and Radiation Oncology

Senior Scientist and Clinical Physicist, Princess Margaret Cancer Center ${ }^{5}$; Head of the Laboratory of Optical Coherence Tomography';

N.D. Gladkova, MD, DSc, Professor, Head of the Laboratory for Studies of Optical Structure of Biotissues, Institute of Biomedical Technologies ${ }^{1}$

${ }^{1}$ Privolzhsky Research Medical University, 10/1 Minin and Pozharsky Square, Nizhny Novgorod, 603005, Russia;

${ }^{2}$ Institute of Applied Physics, Russian Academy of Sciences, 46 Ulyanova St., Nizhny Novgorod, 603155, Russia;

${ }^{3}$ University of Toronto, 101 College St., Toronto, ON M5G 1L7, Canada;

${ }^{4}$ University of Toronto, 150 College St., Toronto, ON M5S 3E2, Canada;

${ }^{5}$ University Health Network, 610 University Avenue, Toronto, ON M5G 2M9, Canada

Determining boundaries of infiltrative glial tumors remains a challenging problem in neurooncology. Optical coherence tomography (OCT) with cross-polarization (CP) visualization is a promising technique as a surgical guidance tool. However, the outcome of the procedures performed under OCT guidance strongly depends on the surgeon's qualification. Thus, a quantitative method for assessing resection margins with OCT is required.

The aim of this study was to develop a robust quantitative approach for CP OCT data to differentiate tumorous from non-tumorous tissues in a rat glioma model.

Materials and Methods. The study was carried out on the rats' brains $(n=6)$ with C6-glioma model injected into the right hemisphere. The left hemisphere was used as a control. The spectral domain CP OCT device that provides two images: in co- and cross-polarizations was used in the study. The central wavelength of probing light was $1310 \mathrm{~nm}$, a spectral width of $100 \mathrm{~nm}$, resulting in axial resolution of $10 \mu \mathrm{m}$. The lateral resolution is $15 \mu \mathrm{m}$. CP OCT images were collected ex vivo with non-contact forward-looking probe after brain excision and its sagittal crosscutting. A total of five CP OCT data sets were collected from each rat brain over the $2.4 \times 2.4 \times 1.25 \mathrm{~mm}$ OCT imaging volumes at the following locations: in the right hemisphere with C6-glioma at the center of the tumor site, at the tumor-non-tumor (white matter) margin; in the contralateral hemisphere of the brain (control) at a selected non-cancer sites: grey matter, gray-white matter margin and visually normal white matter. Quantitative assessment of the different tissue types was based on calculating three optical coefficients:

Corresponding author: Elena B. Kiseleva, e-mail: kiseleva84@gmail.com 
backscattering coefficients relation, attenuation coefficient, and forward cross-scattering coefficient. The CP OCT scanned tissue sites were marked and underwent histological verification.

Results. Color-coded maps of three optical coefficients for normal grey matter and its margin with white matter, C6-glioma and its margin with white matter were generated. Color-coded maps look more representative in margin detection in comparison with en-face $\mathrm{CP}$ OCT images. Every coefficient can differentiate white matter from other tissue types. Comparison of C6-glioma and gray matter color-coded maps reveal poor differentiation capability between these tissues.

Conclusion. A method for 3D CP OCT data quantification using co- and cross-scattering was developed and applied to OCT data volumes. Obtained values were plotted as color-coded maps of different brain tissues and brain tumor. A more accurate determination of tumor margins is obtained using processed CP OCT images in comparison with unprocessed initial images.

Key words: brain white matter; cortex; glioma model; tumor margin; cross-polarization optical coherence tomography; CP OCT; backscattering; attenuation coefficient; cross-scattering.

How to cite: Kiseleva E.B., Yashin K.S., Moiseev A.A., Snopova L.B., Gelikonov G.V., Medyanik I.A., Kravets L.Ya., Karyakin N.N., Vitkin I.A., Gladkova N.D. Quantitative cross-polarization optical coherence tomography detection of infiltrative tumor margin in a rat glioma model: a pilot study. Sovremennye tehnologii v medicine 2018; 10(1): 6, https://doi.org/10.17691/stm2018.10.1.01

\section{Introduction}

Gliomas are the most common tumors (34\%) of central nervous system in adult population [1, 2]. Unfortunately, despite substantial advances in the medical treatment of gliomas in recent years, there is still no great progress in survival [3]. Surgery remains the milestone of the treatment, and its main paradigm is maximum resection while minimizing the risk of damaging eloquent brain areas [4]. The extent of the tumor resection plays a major role as an independent factor associated with improved overall and progression-free survival, most significantly for low-grade gliomas [4-11]. However, total removal of tumors is difficult due to their infiltrative growth into surrounding brain parenchyma. Thus, gross total tumor resection using conventional white-light microscopy can be achieved only in $25-30 \%$ of cases [7, 8, 12]. Assistive technologies such as fluorescence imaging and intraoperative MRI significantly enhance opportunities for the neurosurgeon and increase survival rate [13-16]. However, these methods also have some limitations such as necessity of using contrast agents and lack of sufficient resolution. Thus, optical bioimaging techniques such as multiphoton tomography [17] and optical coherence tomography $(\mathrm{OCT})[18,19]$ providing spatial resolutions in the range from 1 to $20 \mu \mathrm{m}$ and requiring no external contrast look promising for a more precise detection of infiltrative tumor and also can be considered as methods of optical biopsy.

Optical coherence tomography is based on lowcoherence interferometry in the near IR range of wavelengths $(700-1300 \mathrm{~nm})$ to image tissue microstructure in real-time and up to micron resolution at the depths of 1-2 mm [20]. Recently, OCT was used for intraoperative identification of tumor margins in the surgical treatment of glial brain tumors using handled probe $[21,22]$ or microscope integrated OCT systems $[23,24]$. A number of ex vivo and in vivo studies have shown the capacity of OCT for differentiating tumorous and non-tumorous tissues through qualitative [21] and quantitative [25] assessment of the OCT signal. Quantitative assessment is based on optical coefficients calculation, mainly the attenuation coefficient [21, 25, 26] and enhances diagnostic accuracy. An en-face colorcoded attenuation maps construction is another approach for volumetric OCT data processing $[21,26]$ to delineate cancer and non-cancer regions. Application of this mode intraoperatively seems to be very convenient for neurosurgeons.

Traditionally OCT has been developed for stratified tissue types, for example retina [27], gastrointestinal [28] and urinary bladder wall [29]. In visualization of structureless tissues, such as brain tissue, polarizationsensitive OCT appears to be more informative than traditional OCT, because registration of the effect of birefringence from myelinated nerve fibers can help to identify normal white matter and determine orientation in the white matter tracts [30, 31]. Polarization-sensitive OCT is widely used for mapping fiber orientations and tractography in the brain. Cross-polarization OCT (CP OCT) is another polarization-sensitive technique that can image of the initial polarization state changes both due to birefringence and cross-scattering [32, 33]. Previous studies have demonstrated well-defined differences between tumorous and non-tumorous tissues in CP OCT images [34, 35]. This work presents new possibilities for creating color-coded optical coefficients maps of grey matter, white matter, and C6-glioma infiltrative zone based on CP OCT data.

\section{Materials and Methods}

Rat model. The study was carried out on rats' brains with C6-glioma model injected into the right hemisphere at the dose of $1 \cdot 10^{5}$ cells $/ 20 \mu$ of cultural medium. The left hemisphere was used as a control. Animals (female Wistar rats aged $8 \pm 10$ weeks, $n=6$ ) with gliomas were purchased from Research Institute of Human Morphology, Moscow, Russia. C6-glioma is a chemically induced brain tumor (Grade IV) that is morphologically 
similar to human glioblastoma (high cellular density, cell polymorphism, presence of necrotic and hemorrhage areas, infiltrative growth). CP OCT imaging was performed on days 17-19 after the transplantation of glioma, when the tumor had formed and was visible on the brain surface (Figure 1 (b)).

All animal experimentations met the requirements described in Rules for the Work using Experimental Animals (Russia, 2010) and International Guiding Principles for Biomedical Research Involving Animals (CIOMS and ICLAS, 2012); the ethical principles established by the European Convention for the Protection of Vertebrate Animals used for Experimental and Other Scientific Purposes (Strasbourg, 2006) were strictly observed. The studies were approved by the Ethics Committee of the Nizhny Novgorod State Medical Academy (protocol No.14 from 10 December 2013).

Cross-polarization optical coherence tomography device. The spectral domain $\mathrm{CP}$ OCT device that provides two image acquisition in co- and crosspolarization was used in the study. A detailed description of the system including the optical layout and crosspolarization image registration has been published previously [36, 37]. In brief, the light source of the CP OCT device is a superluminescent diode with a central wavelength of $1310 \mathrm{~nm}$, a spectral width of $100 \mathrm{~nm}$, resulting in axial resolution of $10 \mu \mathrm{m}$. The lateral resolution was $15 \mu \mathrm{m}$. CP OCT images were collected ex vivo using non-contact forward-looking probe (Figure 2 (a)). Acquisition rate was 20,000 A-scans/s, frequency of $B$-scans registration for each pair of images with a size of $2.4 \times 1.25 \mathrm{~mm}-40$ shots/s. Total acquisition time of $3 \mathrm{D}$ data set was $26 \mathrm{~s}$.

Study design. This study evaluates feasibility of CP OCT in delineating tumorous from non-tumorous tissue on rat C6-glioma model. 3D CP OCT images of both hemispheres (Figure 2 (b)) were acquired ex vivo after brain excision and its sagittal crosscutting (see Figure 2 (a)): right hemisphere - through the visual tumor center, left hemisphere - at the same plane as the right one for a more accurate comparison (Figure 1). A total of five CP OCT data sets were collected from each rat brain over the
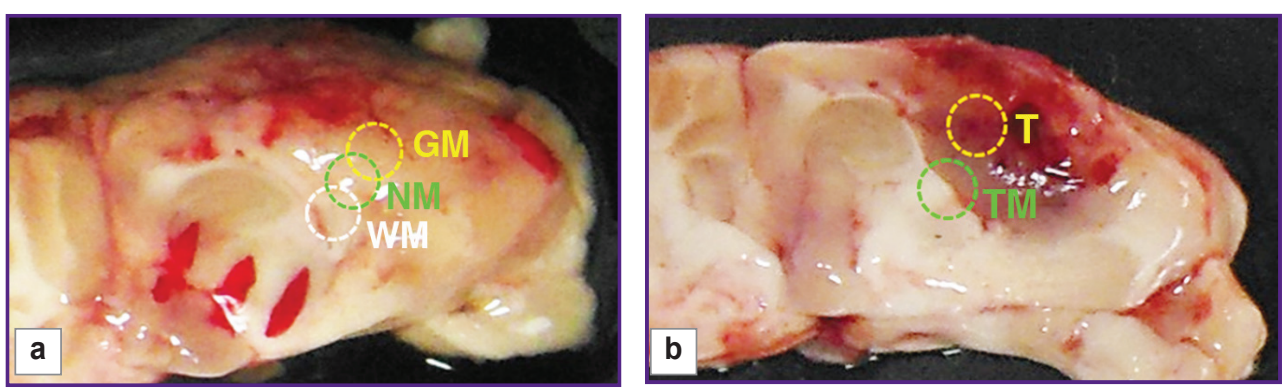

Figure 1. Regions of interest for CP OCT study of rat brain:

(a) left (control) hemisphere; GM - grey matter, NM - normal tissues margin, WM - white matter; (b) right (with C6-glioma) hemisphere; T — tumor, TM - tumor margin. Sagittal crosscutting was done through the center of each hemisphere. Red marks in (a) indicate the direction of the CP OCT scanning

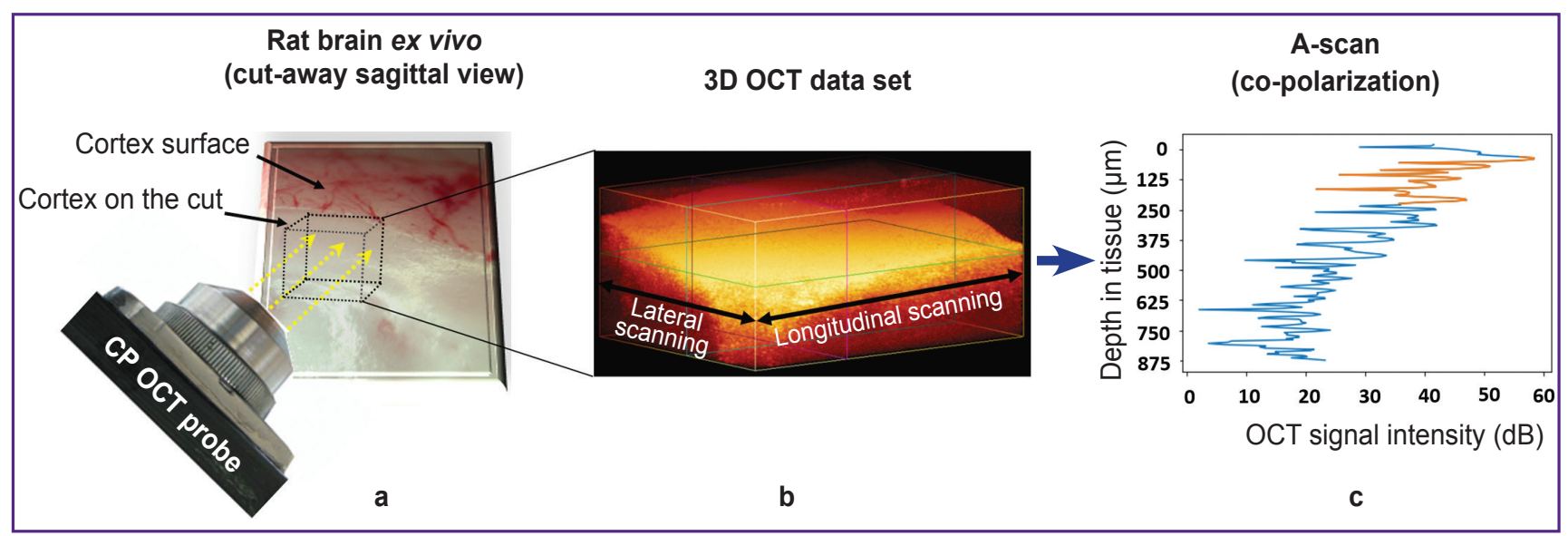

Figure 2. Scheme of the CP OCT study of rat brain:

(a) the cut-away sagittal view of the brain, the yellow dashed arrows indicate that the scan occurs from the side of the cut (from the inside) of the brain; (b) 3D OCT image consisting of a set of A-scans; (c) randomly taken A-scan, orange area indicates the depth range $(50-250 \mu \mathrm{m})$, in which optical coefficients were calculated 
$2.4 \times 2.4 \times 1.25 \mathrm{~mm}$ OCT imaging volumes at the following locations (regions of interest): in the right hemisphere with C6-glioma at the center of the tumor site, at the tumor-non-tumor (white matter) margin (see Figure 1 (b)); in the contralateral hemisphere of the brain (control) at selected non-cancer sites - grey matter, gray-white matter margin, and visually normal white matter (Figure 1 (a)). 3D CP OCT images of the left hemisphere were collected with some overlap. Each imaging data set consisted of $256 \times 208$ A-scans forming OCT imaging volume. For each collected A-scan set, optical coefficients were calculated in the predefined depth range according to the method described in the corresponding subsection "Optical coefficients estimation". After CP OCT scanning, all regions of interest were marked and consequently correlated with histology.

Optical coefficients estimation. Since visualized brain tissue is optically uniform at the OCT imaging depth, in order to fully utilize information content in two acquired orthogonal polarizations, polarization effects were described with three coefficients additionally to conventionally used attenuation coefficient and a method of their estimation was developed. These coefficients describe the rate of back and forward scattering in polarization, which is orthogonal to incident state. Birefringence of white matter (irregular birefringence on myelinated nerve fibers) was considered in the calculations as cross-scattering.

For derivation of dependencies governing light propagation in turbid media with respect to crosspolarization scattering, particle balance equations were formed and solved for the photons of scanning beam propagating through the tissue. The following possibilities for propagating photon were considered:

1. Photon propagating in the initial polarization backscatters in the same polarization and is being detected. The number of such events is proportional to the light intensity at a certain depth with backscattering coefficient $\mu$.

2. Photon propagating in initial polarization backscatters in the orthogonal polarization and is being detected. The number of such events is proportional to the light intensity at certain depth with cross-scattering coefficient $\mu_{x}$.

3. Photon propagating in initial polarization scatters in forward direction in the orthogonal polarization and further propagates as the light in orthogonal polarization. The number of such events is proportional to the light intensity at certain depth with forward cross-scattering coefficient $C$.

4. Photon propagating in initial polarization scatters sideways or is being detected by the tissue. The number of such events is proportional to the light intensity at certain depth with attenuation coefficient $v$.

Since utilized scanning beam had circular polarization, conversion rates between orthogonal polarizations were considered equal.

Accounting for all the aforementioned processes, the scanning beam intensity changes with depth $z$ can be written as:

$$
\begin{aligned}
& \frac{\mathrm{d} I_{c o}}{\mathrm{~d} z}=-\left(\mu+\mu_{x}+v+C\right) I_{c o}+C \cdot I_{\text {cross }} ; \\
& \frac{\mathrm{d} l_{\text {cross }}}{\mathrm{d} z}=-\left(\mu+\mu_{\mathrm{x}}+v+C\right) I_{\text {cross }}+C \cdot I_{\text {co }} .
\end{aligned}
$$

Here $I_{c o}$ - scanning beam intensity in initial polarization, $I_{\text {cross }}$ - scanning beam intensity in orthogonal polarization. Solving system of equations (1) with initial conditions $I_{c o}(0)=I_{0} \quad$ (where $I_{0}$ — initial scanning beam intensity), $I_{\text {cross }}(0)=0$, one can get:

$$
\begin{aligned}
& I_{c o}=\frac{I_{0}}{2} \exp \left[-\left(\mu+\mu_{x}+v\right) z\right]+\frac{I_{0}}{2} \exp \left[-\left(\mu+\mu_{x}+v+2 C\right) z\right] \\
& I_{\text {cross }}=\frac{I_{0}}{2} \exp \left[-\left(\mu+\mu_{x}+v\right) z\right]-\frac{I_{0}}{2} \exp \left[-\left(\mu+\mu_{x}+v+2 C\right) z\right] .
\end{aligned}
$$

Following the same logic, changes of cumulated light power detected in each channel can be written as:

$$
\begin{aligned}
& \frac{\mathrm{d} S_{c o}}{\mathrm{~d} z}=\mu \cdot I_{c o}+\mu_{x} \cdot I_{\text {cross }} ; \\
& \frac{\mathrm{d} S_{\text {cross }}}{\mathrm{d} z}=\mu \cdot I_{\text {cross }}+\mu_{x} \cdot I_{c o} .
\end{aligned}
$$

Here $S_{c o}$ - cumulated light power detected in initial polarization, $S_{\text {cross }}$ - cumulated light power detected in orthogonal polarization. Note that derivatives in (3) are actually detected powers in the corresponding channels at the depth $z$.

Plugging expressions (2) into equations (3), then adding and subtracting resulting equations one can get:

$$
\begin{aligned}
& \frac{\mathrm{d} S_{c o}}{\mathrm{~d} z}+\frac{\mathrm{d} S_{\text {cross }}}{\mathrm{d} z}=I_{0}\left(\mu+\mu_{x}\right) \exp \left[-\left(\mu+\mu_{x}+v\right) z\right] \\
& \frac{\mathrm{d} S_{c o}}{\mathrm{~d} z}-\frac{\mathrm{d} S_{\text {cross }}}{\mathrm{d} z}=I_{0}\left(\mu-\mu_{x}\right) \exp \left[-\left(\mu+\mu_{x}+v\right) z\right] .
\end{aligned}
$$

Division of equations (4) is free from unknown constant $I_{0}$ :

$$
\frac{d\left(S_{c o}+S_{c r o s s}\right)}{d z}: \frac{d\left(S_{c o}-S_{c r o s s}\right)}{d z}=\frac{\mu+\mu_{x}}{\mu-\mu_{x}} \exp (2 C z) \text {. }
$$

Since investigated tissue samples are assumed to be optically uniform, linear approximation of the logarithm of division of sum and difference of the signals of both channels permits to obtain values of $\mu_{x} / \mu$ and $C$ from equation (5). Linear term coefficients from linear approximations of sum and difference of the signals in both channels permit to obtain value of coefficient $v$ according to equation (4). Thus three measured optical coefficients are: backscattering coefficients relation $\mu_{x} / \mu$, attenuation coefficient $v$, and forward cross-scattering coefficient $C$.

Histology. The CP OCT scanned regions of interests were marked with histological ink, samples were fixed in $10 \%$ formalin for $48 \mathrm{~h}$ and a series of histological sections through the marked area were done. Thus, the plane of CP OCT scanning coincided equidistantly with the plane of the histological sections. Histological sections 
were stained with hematoxylin and eosin and were observed in transmitted light with a Leica DM2500 (Leica Microsystems, Germany) microscope, equipped with a DFC 245C digital camera.

For each of the histological sections we recorded the characteristics of the tissue structure, grouping them as follows: group 1 - normal grey matter; group 2 - normal white matter; group 3 - C6-glioma; group 4 - grey matter-white matter margin; group 5 - C6-glioma-white matter margin.

\section{Results}

Quantitative assessment of the different tissue type was based on calculating three optical coefficients for cortex (grey matter), white matter, and C6-glioma states as well as for specimens containing borders between different tissue types (grey matter-white matter, tumorwhite matter). Color-coded 2D maps of optical coefficients were constructed for the latter.

Based on histopathology data, 3 groups of 3D CP OCT images were formed and quantitatively assessed: normal grew matter (group 1), normal white matter (group 2), and tumor only (group 3). Histograms of three optical coefficients - backscattering coefficients relation, attenuation coefficient, and forward cross-scattering coefficient for white matter, gray matter, and glioma tissue are plotted in Figure 3. White matter mostly consists of densely packed elongated elements (myelinated nerve fibers randomly arranged under the cortex — Figure 4 (a2)), which leads to higher backscattering as well as to more prominent polarization effects (as seen from Figure 3, blue curve) in comparison with gray matter (Figure 4 (a1)) and glioma tissue (Figure 5 (a1)) which

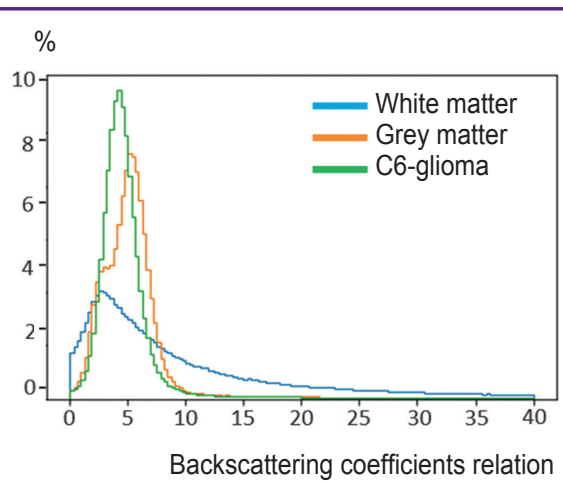

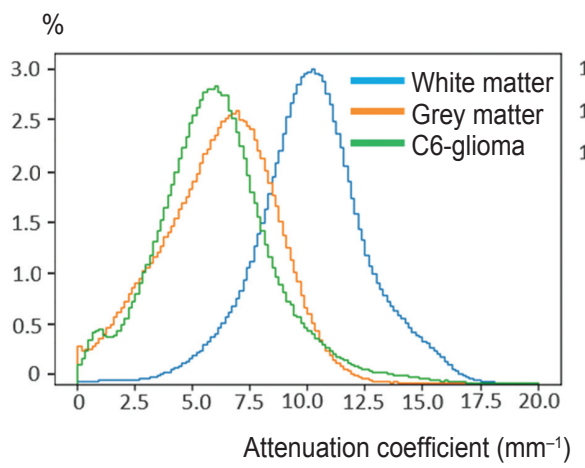

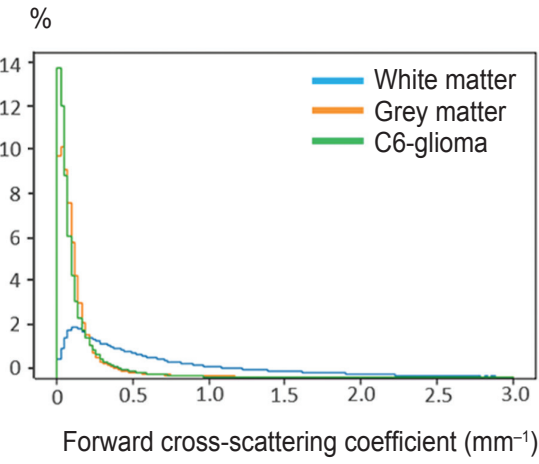

Figure 3. Optical coefficients histograms for different tissue types: white matter (b/ue), grey matter (orange), and C6-glioma (green):

(a) backscattering coefficients relation; (b) attenuation coefficient; (c) forward cross-scattering coefficient
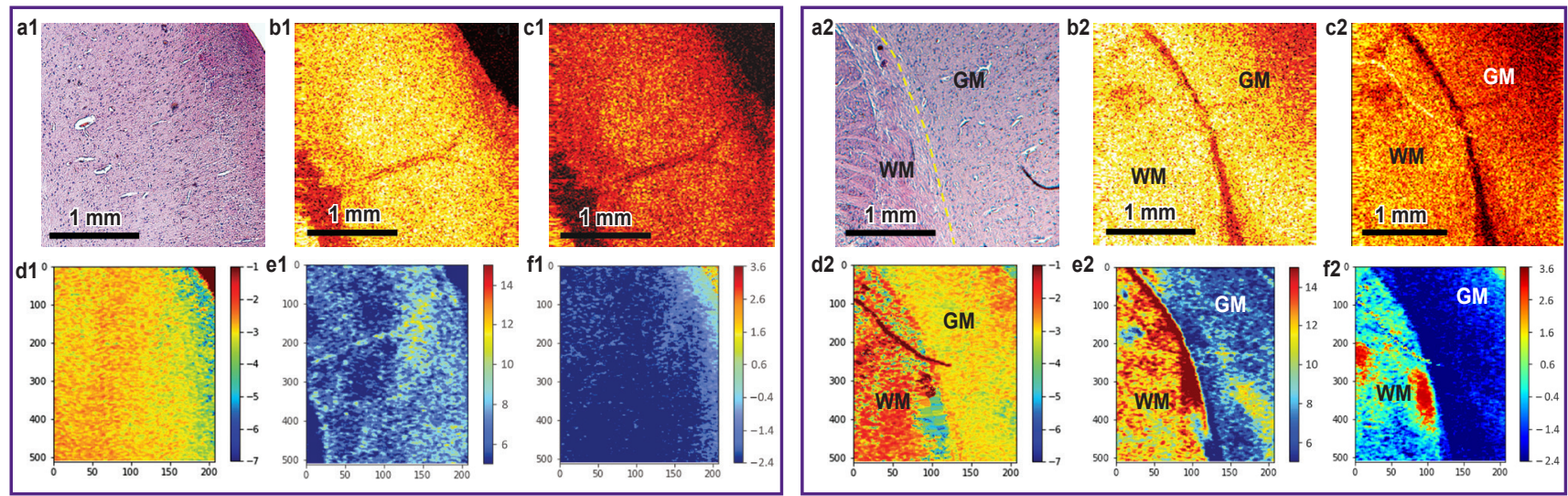

Figure 4. Initial and processed en-face CP OCT images of normal brain tissues and their margins

Unprocessed initial en-face CP OCT images of grey matter in co- (b1) and cross- (c1) polarizations, grey matter-white matter margin in co- (b2) and cross- (c2) polarizations and corresponding color-coded maps of grey matter (d1), (e1), (f1), grey matter-white matter margin (d2), (e2), (f2). Optical coefficients: (d1), (d2) backscattering coefficients relation in log scale; (e1), (e2) attenuation coefficient; (f1), (f2) forward cross-scattering coefficient in log scale. Histological images (a1), (a2), hematoxylin and eosin staining. GM - grey matter, WM — white matter 

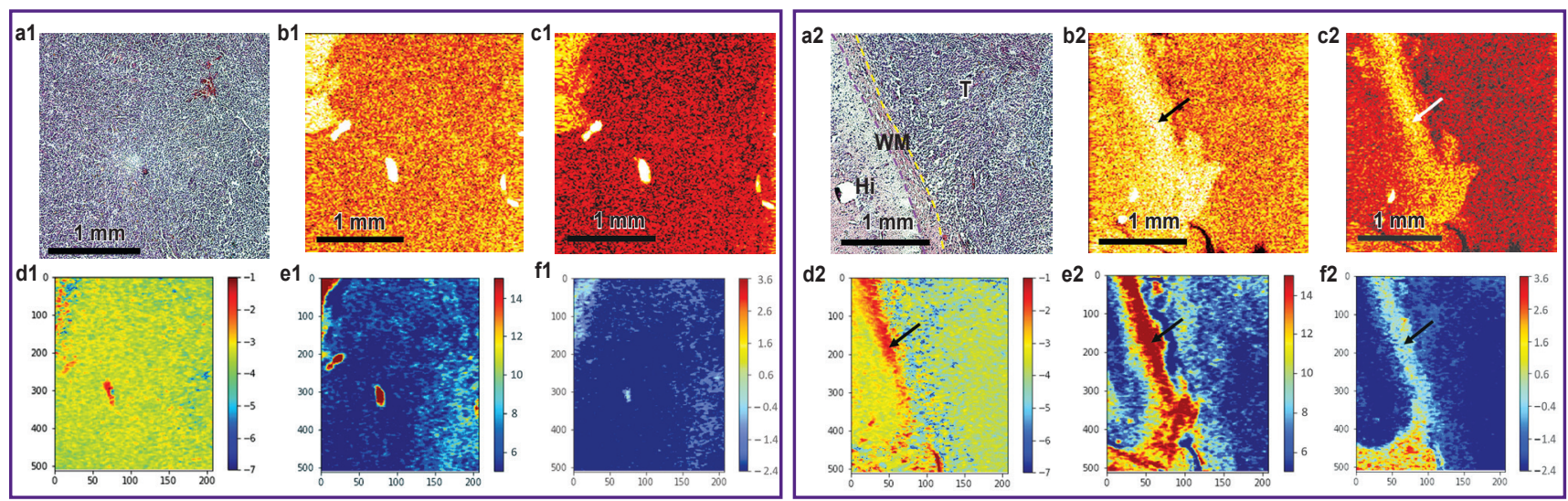

Figure 5. Initial and processed en-face CP OCT images of tumorous-non-tumorous brain tissues and its margin Unprocessed initial en-face CP OCT images of C6-glioma in co- (b1) and cross- (c1) polarizations, C6-glioma-white matter margin in co- (b2) and cross- (c2) polarizations and corresponding color-coded maps of C6-glioma (d1), (e1), (f1), C6-glioma-white matter margin (d2), (e2), (f2). Optical coefficients: (d1), (d2) backscattering coefficients relation in log scale; (e1), (e2) attenuation coefficient; (f1), (f2) forward cross-scattering coefficient in log scale. Histological images (a1), (a2), hematoxylin and eosin staining. $\mathrm{Hi}$ - hippocampus, WM — white matter, $\mathrm{T}$ - tumor. Arrows indicate white matter

consist mostly of round-like structures (cells) with loose spatial arrangement and do not manifest pronounced backscattering and polarization properties (as seen from Figure 3, green and orange curves). While attenuation coefficient provides clear differentiating for white matter from gray matter and glioma tissue (Figure 3 (b)), 2D distribution of polarization-related coefficient may form specific appearance of white matter due to higher standard deviation of the coefficients distribution (Figure 3 (c)).

Optical coefficients color-coded maps of normal brain tissues and their margins. Color-coded maps of normal grey matter and its margin with white matter, corresponding en-face CP OCT images and histology are presented in Figure 4. Grey matter and white matter could be differentiated by each of suggested coefficient. Visually it is well-defined on color-coded maps which are generated across the grey matter-white matter margin (Figure 4 (d2)-(f2)).

Optical coefficients color-coded maps of tumorous-non-tumorous brain tissues and their margins. Color-coded maps of C6-glioma and its margin with white matter, corresponding en-face $\mathrm{CP}$ OCT images and histology are presented in Figure 5. Similar to previously described differences between grey matter and white matter, the white matter and C6-glioma could also be distinguished by optical coefficients. In Figure 5 (d2)-(f2), the white matter forms a narrow band (arrows) which is seen in red (d2), (e2) and light blue (f2) color. The left part of the image represents brain basal structures (hippocampus). Note, that these structures are better differentiated from tumor tissue in the right side of the image in backscattering coefficients relation (d2) while showing almost identical features in attenuation coefficient channel (e2).

Visually, every coefficient differentiates white matter and other tissue types. Color-coded maps look more representative in margin detection in comparison to enface CP OCT images.

\section{Discussion}

Infiltrative growth of brain tumors causes difficulty in achieving gross total tumor resection. An introduction of innovative minimally invasive methods like OCT for intraoperative guidance appears to be a critical challenge for the modern brain tumor surgery. Various approaches for qualitative evaluation of OCT images and scoring algorithms for gliomas/normal differentiation criteria have been actively developed [21, 25, 26], but still remain an actual scientific task. Kut et al. [21] demonstrated that attenuation coefficient estimated from 3D OCT images could be used to quantitatively differentiate between tumorous and non-tumorous tissues and help guide brain tumor resections. A color-coded map denoting regions of high and low attenuation, corresponding to glial tumor and normal tissue, respectively, was generated to provide visual feedback to the surgeon. The system's algorithm was developed using ex vivo human tissues and tested during in vivo mouse surgery. However, direct transfer of established in ex vivo human samples optimal attenuation threshold to in vivo mouse brain with glial tumor model needs to be clarified.

CP OCT functionality lies between polarizationsensitive microscopic OCT and OCT without polarization sensitivity. Although it does not provide orientation of each myelin fiber, it is sensitive to cumulative polarization effects which lead to cross-scattering.

In this study, a method for obtaining three optical coefficients was developed using OCT images in co- and cross-scattering channels for more contrast detection of glial tumor margin. CP OCT can differentiate white matter 
and glial tumor, which have certain dominant values on color-coded maps. In addition, presence of clearly visible delineation between white matter and grey matter can be potentially used in stereotactic functional neurosurgery for precise identification basal ganglia (for example, subthalamic nucleus which is surrounded by white matter tracts). There is a problem of poor differentiation capability between C6-glioma and grey matter, which both have low coefficients values and look similar on the colorcoded maps. It can cause difficulties in determination of tumor margin along basal structures of grey matter (e.g., thalamus) in deep-seated glioma. Due to that, it offers challenge to suggest combination of optical coefficients and optimize the CP OCT numerical processing procedure which will be the next step of our research.

\section{Conclusions}

A method for 3D CP OCT data quantification using coand cross-scattering images was developed and applied to images of tumorous and non-tumorous brain tissues. Color-coded maps of proposed coefficients distribution in different brain tissues and brain tumor were constructed. Contrast of infiltrative tumor margin with white matter on color-coded maps was demonstrated. A more accurate determination of tumor margins is obtained using processed CP OCT images in comparison with unprocessed initial images.

Acknowledgements. The experimental part of this work, as well as the numerical image processing, analysis of the efficiency of algorithms for image quantification and selection of optimal quantitative characteristics, were supported by the Russian Scientific Foundation grant No.16-15-10391 (EBK, KSYa, LBS, NDG). The method for optical coefficients calculation was supported by Russian Foundation for Basic Research project No.16-3260178 mol_a_dk (AAM).

Conflict of interest. The authors declare they have no conflicts of interest that need to be reported.

\section{References}

1. Kohler B.A., Sherman R.L., Howlader N., Jemal A., Ryerson A.B., Henry K.A., Boscoe F.P., Cronin K.A., Lake A., Noone A.-M., Henley S.J., Eheman C.R., Anderson R.N., Penberthy L. Annual Report to the Nation on the Status of Cancer, 1975-2011, featuring incidence of breast cancer subtypes by race/ethnicity, poverty, and state. J Natl Cancer Inst 2015; 107(6): djv048, https://doi.org/10.1093/jnci/djv048.

2. Ostrom Q.T., Gittleman H., Farah P., Ondracek A., Chen Y., Wolinsky Y., Stroup N.E., Kruchko C., BarnholtzSloan J.S. CBTRUS statistical report: primary brain and central nervous system tumors diagnosed in the United States in 2006-2010. Neuro Oncol 2013; 15(Suppl 2): ii1-ii56, https:// doi.org/10.1093/neuonc/not151.

3. Lichtenberg F.R. The expanding pharmaceutical arsenal in the war on cancer. National Bureau of Economic Research, Working Paper Series 2004; 10328, https://doi.org/10.3386/ w10328.
4. Almeida J.P., Chaichana K.L., Rincon-Torroella J., Quinones-Hinojosa A. The value of extent of resection of glioblastomas: clinical evidence and current approach. Curr Neurol Neurosci Rep 2014; 15(2): 517, https://doi.org/10.1007/ s11910-014-0517-x.

5. Sanai N., Berger M.S. Glioma extent of resection and its impact on patient outcome. Neurosurgery 2008; 62(4): 753766, https://doi.org/10.1227/01.neu.0000318159.21731.cf.

6. Sanai N., Polley M.Y., McDermott M.W., Parsa A.T., Berger M.S. An extent of resection threshold for newly diagnosed glioblastomas $J$ Neurosurg 2011; 115(1): 3-8, https://doi.org/10.3171/2011.2.jns10998.

7. Reulen H.J., Meinel T., Pichlmeier U., Schumacher W., Tonn J.C., Rohde V., Oppel F., Turowski B., Woiciechowsky C., Franz K., Pietsch T; ALA-Glioma Study Group. Extent of resection and survival in glioblastoma multiforme: identification of and adjustment for bias. Neurosurgery 2008; 62(3): 564576, https://doi.org/10.1227/01.neu.0000317304.31579.17.

8. McGirt M.J., Chaichana K.L., Gathinji M. Attenello F.J., Than K., Olivi A., Weingart J.D., Brem H., Quiñones-Hinojosa A.R. Independent association of extent of resection with survival in patients with malignant brain astrocytoma. J Neurosurg 2009; 110(1): 156-162, https://doi. org/10.3171/2008.4.17536

9. McGirt M.J., Chaichana K.L., Attenello F.J., Weingart J.D., Than K., Burger P.C., Olivi A., Brem H., Quinoñes-Hinojosa A. Extent of surgical resection is independently associated with survival in patients with hemispheric infiltrating low-grade gliomas. Neurosurgery 2008; 63(4): 700-708, https://doi.org/10.1227/01. neu.0000325729.41085.73.

10. Sanai N., Berger M.S. Extent of resection influences outcomes for patients with gliomas. Rev Neurol (Paris) 2011; 167(10): 648-654, https://doi.org/10.1016/j. neurol.2011.07.004.

11. Lacroix M., Abi-Said D., Fourney D.R., Gokaslan Z.L., Shi W., DeMonte F., Lang F.F., McCutcheon I.E., Hassenbusch S.J., Holland E., Hess K., Michael C., Miller D., Sawaya R. A multivariate analysis of 416 patients with glioblastoma multiforme: prognosis, extent of resection, and survival. J Neurosurg 2001; 95(2): 190-198, https://doi. org/10.3171/jns.2001.95.2.0190.

12. Colditz M.J., Jeffree R.L. Aminolevulinic acid (ALA)protoporphyrin IX fluorescence guided tumour resection. Part 1: Clinical, radiological and pathological studies. J Clin Neurosci 2012; 19(11): 1471-1474, https://doi.org/10.1016/j. jocn.2012.03.009.

13. Zhao S., Wu J., Wang C., Liu H., Dong X., Shi C., Shi C., Liu Y., Teng L., Han D., Chen X., Yang G., Wang L., Shen $\mathrm{C}$., Li H. Intraoperative fluorescence-guided resection of high-grade malignant gliomas using 5-aminolevulinic acidinduced porphyrins: a systematic review and meta-analysis of prospective studies. PLoS One 2013; 8(5): e63682, https://doi. org/10.1371/journal.pone.0063682.

14. Eljamel S. 5-ALA fluorescence image guided resection of glioblastoma multiforme: a meta-analysis of the literature. Int J Mol Sci 2015; 16(5): 10443-10456, https://doi. org/10.3390/ijms160510443.

15. Kubben P.L., ter Meulen K.J., Schijns O.E., ter LaakPoort M.P., van Overbeeke J.J., van Santbrink H. Intraoperative MRI-guided resection of glioblastoma multiforme: a systematic review. Lancet Oncol 2011; 12(11): 1062-1070, https://doi. org/10.1016/s1470-2045(11)70130-9. 
16. Suero Molina E., Schipmann S., Stummer W. Maximizing safe resections: the roles of 5-aminolevulinic acid and intraoperative MR imaging in glioma surgery - review of the literature. Neurosurg Rev 2017; 18, https://doi.org/10.1007/ s10143-017-0907-z.

17. Kantelhardt S.R., Kalasauskas D., König K., Kim E., Weinigel M., Uchugonova A., Giese A. In vivo multiphoton tomography and fluorescence lifetime imaging of human brain tumor tissue. J Neurooncol 2016; 127(3): 473-482, https://doi. org/10.1007/s11060-016-2062-8.

18. Vasefi F., MacKinnon N., Farkas D.L., Kateb B. Review of the potential of optical technologies for cancer diagnosis in neurosurgery: a step toward intraoperative neurophotonics. Neurophotonics 2016; 4(1): 011010, https:// doi.org/10.1117/1.nph.4.1.011010.

19. Yashin K.S., Kravets L.Y., Gladkova N.D., Gelikonov G.V., Medyanik I.A., Karabut M.M., Kiseleva E.B., Shilyagin P.A. Optical coherence tomography in neurosurgery. Voprosy neirokhirurgii imeni N.N. Burdenko 2017; 81(3): 107115, https://doi.org/10.17116/neiro2017813107-115.

20. Huang D., Swanson E.A., Lin C.P., Schuman J.S., Stinson W.G., Chang W., Hee M.R., Flotte T., Gregory K., Puliafito C.A., Fujimoto J.G. Optical coherence tomography. Science 1991; 254(5035): 1178-1181, https://doi.org/10.1126/ science.1957169.

21. Kut C., Chaichana K.L., Xi J., Raza S.M., Ye X., McVeigh E.R., Rodriguez F.J., Quinones-Hinojosa A., Li X. Detection of human brain cancer infiltration ex vivo and in vivo using quantitative optical coherence tomography. Sci Transl Med 2015; 7(292): 292ra100-292ra100, https://doi. org/10.1126/scitranslmed.3010611.

22. Sun C., Lee K. K.C., Vuong B., Cusimano M., Brukson A., Mariampillai A., Standish B.A., Yang V.X.D. Neurosurgical hand-held optical coherence tomography (OCT) forward-viewing probe. Photonic Therapeutics and Diagnostics VIII 2012; 82074V, https://doi.org/10.1117/12.909116.

23. Lankenau, E., Klinger D., Winter C., Malik A., Müller H.H., Oelckers S., Pau H.-W., Just T., Hüttmann G. Combining optical coherence tomography (OCT) with an operating microscope. Advances in Medical Engineering 2007; 114: 343-348, https://doi.org/10.1007/978-3-540-68764-1_57.

24. El-Haddad M.T., Tao Y.K. Advances in intraoperative optical coherence tomography for surgical guidance. Biomed Opt Express 2017; 3: 37-48, https://doi.org/10.1016/j. cobme.2017.09.007.

25. Böhringer H.J., Lankenau E., Stellmacher F., Reusche E., Hüttmann G., Giese A. Imaging of human brain tumor tissue by near-infrared laser coherence tomography. Acta Neurochir (Wien) 2009; 151(5): 507-517, https://doi. org/10.1007/s00701-009-0248-y.

26. Yuan W., Kut C., Liang W., Li X. Robust and fast characterization of OCT-based optical attenuation using a novel frequency-domain algorithm for brain cancer detection. Sci Rep 2017; 7: 44909, https://doi.org/10.1038/srep44909.

27. Al Kharousi N., Wali U.K., Azeem S. Chapter 1.
Current applications of optical coherence tomography in ophthalmology. In: Optical coherence tomography. Edited by Kawasaki M. InTech; 2013, https://doi.org/10.5772/53961.

28. Tsai T.-H., Leggett C.L., Trindade A.J., Sethi A., Swager A.-F., Joshi V., Bergman J.J., Mashimo H., Nishioka N.S., Namati E. Optical coherence tomography in gastroenterology: a review and future outlook. J Biomed Opt 2017; 22(12): 121716, https://doi.org/10.1117/1.jbo.22.12.121716.

29. Wang H.-W., Chen Y. Clinical applications of optical coherence tomography in urology. Intravital 2014; 3(1): e28770, https://doi.org/10.4161/intv.28770.

30. Wang H., Akkin T., Magnain C., Wang R., Dubb J., Kostis W.J., Yaseen M.A., Cramer A., Sakadžić S., Boas D. Polarization sensitive optical coherence microscopy for brain imaging. Opt Lett 2016; 41(10): 2213-2216, https://doi. org/10.1364/ol.41.002213.

31. Boas D.A., Wang H., Magnain C., Fischl B. Polarization-sensitive optical coherence tomography of the human brain connectome. SPIE Newsroom 2017, https://doi. org/10.1117/2.1201701.006834.

32. Gubarkova E.V., Dudenkova V.V., Feldchtein F.I., Timofeeva L.B., Kiseleva E.B., Kuznetsov S.S., Shakhov B.E., Moiseev A.A., Gelikonov G.V., Vitkin A., Gladkova N.D. Multimodal optical imaging characterization of atherosclerotic plaques. J Biophotonics 2016; 9(10): 1009-1020, https://doi. org/10.1002/jbio.201500223.

33. Gladkova N., Kiseleva E., Robakidze N., Balalaeva I., Karabut M., Gubarkova E., Feldchtein F. Evaluation of oral mucosa collagen condition with cross-polarization optical coherence tomography. J Biophotonics 2013; 6(4): 321-329, https://doi.org/10.1002/jbio.201200059.

34. Yashin K.S., Karabut M.M., Fedoseeva V.V., Khalansky A.S., Matveev L.A., Elagin V.V., Kuznetsov S.S., Kiseleva E.B., Kravets L.Y., Medyanik I.A., Gladkova N.D. Multimodal optical coherence tomography in visualization of brain tissue structure at glioblastoma (experimental study). Sovremennye tehnologii v medicine 2016; 8(1): 73-81, https:// doi.org/10.17691/stm2016.8.1.10.

35. Yashin K.S., Gubarkova E., Kiseleva E., Kuznetsov S.S., Karabut M.M., Medyanik I.A., Kravets L.Y., Gladkova N.D. Ex vivo visualization of human gliomas by cross-polarization optical coherence tomography: pilot study. Sovremennye tehnologii v medicine 2016; 8(4): 14-21, https:// doi.org/10.17691/stm2016.8.4.02.

36. Gelikonov V.M., Gelikonov G.V. New approach to erosspolarized optical coherence tomography based on orthogonal arbitrarily polarized modes. Laser Physics Letters 2006: 3(9): 445-451, https://doi.org/10.1002/lapl.200610030.

37. Matveev L.A., Zaitsev V.Y., Gelikonov G.V., Matveyev A.L., Moiseev A.A., Ksenofontov S.Y., Gelikonov V.M., Sirotkina M.A. Gladkova N.D. Demidov V., Vitkin A. Hybrid M-mode-like OCT imaging of three-dimensional microvasculature in vivo using reference-free processing of complex valued B-scans. Opt Lett 2015; 40(7): 1472-1475, https://doi.org/10.1364/ol.40.001472. 